\title{
RATELESS WIRELESS SYSTEMS: GAINS, APPROACHES, AND CHALLENGES
}

\author{
F. Mehran ${ }^{(1)}$, K. Nikitopoulos $^{(1)}$, P. Xiao ${ }^{(1)}, Q$. Chen $^{(2)}$ \\ ${ }^{(1)}$ Institute for Communication Systems (ICS), Home of 5G Innovation Centre, \\ University of Surrey, Guildford, UK. \\ ${ }^{(2)}$ Southwest Jiaotong University, Chengdu, China.
}

\begin{abstract}
State-of-the-art channel coding schemes promise data rates close to the wireless channel capacity. However, efficient link adaptation techniques are required in order to deliver such throughputs in practice. Traditional rate adaptation schemes, which are reactive and try to "predict" the transmission mode that maximizes throughput based on "transmission quality indicators", can be highly inefficient in an evolving wireless ecosystem where transmission can become increasingly dynamic and unpredictable. In such scenarios, "rateless" link adaptation can be highly beneficial. Here, we compare popular rateless approaches in terms of gains and practicality in both traditional and more challenging operating scenarios. We also discuss challenges that need to be addressed to make such systems practical for future wireless communication systems.
\end{abstract}

Index Terms - adaptive modulation and coding (AMC), hybrid automatic repeat request (HARQ), link adaptation, raptor code, rateless.

\section{INTRODUCTION}

The discovery of turbo codes [1] and the re-invention of Low Density Parity Check (LDPC) codes [2],[3] facilitate the performance of communication systems near the capacity limit. However, in order to employ such techniques in real world scenarios, and translate their capabilities into practical throughput gains, link adaptation techniques are required to adjust the transmission rate to the transmission conditions. This adjustment is extensively deployed by Adaptive Modulation and Coding (AMC) which pre-defines combinations of modulation and coding schemes ([4] and references therein).

Failing to choose the correct transmission mode can adversely affect the throughput performance in two ways: i) If the chosen transmission rate is lower than what the link can support, we underutilize the transmission channel and the corresponding capacity. ii) Even worse, if the chosen rate is higher than what the channel can support, the packet delivery will be unsuccessful. In order to moderate the effects of such unsuccessful receptions, Automatic Repeat reQuest (ARQ) schemes are used [5],[6]. In original versions of ARQ schemes, multiple retransmissions of whole erroneous packet were performed, resulting in substantial throughput reduction. To improve the efficiency, enhanced ARQ schemes, known as Hybrid ARQ (HARQ) incorporated channel coding, by transmitting additional parity bits upon receiver's request [7].

A major difference between AMC and HARQ schemes is that, while the former needs to be aware of some kind of "transmission quality indicator" (e.g., channel state information) in advance, HARQ schemes operate blindly. However, and as will be shown in Section 3, current HARQ schemes still require AMC. Rateless systems on the other hand, can fully eliminate AMC. In rateless systems the transmission is initiated using a high transmission rate and then additional parity information is transmitted until the receiver decodes successfully. In this way, the transmission rate is effectively adapted to the temporal channel condition, without requiring any channel feedback from the receiver. An ideal perfect rateless system would, then, be the one that could decode when the effective rate matches the capacity limit.

In this paper, we review some popular rateless approaches, in both traditional and challenging link conditions, and we identify some major challenges that need to be addressed to make such approaches applicable to future generations of wireless communication systems.

\section{RATELESS CODING}

The invention of the first practical rateless paradigm dates back to the breakthrough introduced by Luby Transform (LT) codes [8]. LT code is an erasure correcting code with rateless properties that has been proposed for transmission over erasure channels. Raptor codes were later introduced as the extension of LT codes for attaining capacity of erasure channels [9],[10]. Although rateless codes have been primarily applied for protecting packets against erasures, there have been some extensions considering their performance at the physical layer for protecting data over noisy channels [11],[12]. Applying rateless codes to the physical layer can be advantageous since the decoder can exploit useful information even from packets that cannot be correctly decoded and therefore are ignored by higher layers. 
In [11] and [12], authors elaborated on the performance analysis and comparison of LT and Raptor codes over AWGN channels, and reported a superior performance by Raptor codes. Further evaluations of the performance of Raptor codes over AWGN and fading channels have been conducted in terms of achievable throughputs in [13]. In addition to Raptor codes, other solutions such as Spinal codes [14] and Strider codes [15] have also been introduced. Strider codes, however, show inferior performance than Raptor codes [14] in AWGN channels, while Spinal codes show better performance at the cost of substantial complexity increase. For demonstration purposes, in this paper we will focus on Raptor codes.

While there is a significant amount of work on rateless codes for AWGN channels, limited work exists on rateless approaches for MIMO systems with Spatial Multiplexing (SM-MIMO), and the literature mainly focuses on the aspects of code design [16]-[19]. In Section 4, we present and discuss this topic, and we identify some limitations of the existing approaches.

\section{PERFORMANCE OF RATELESS SYSTEMS IN DIFFERENT PROPAGATION ENVIRONMENTS}

In this section, we examine systems with rateless properties in several transmission scenarios.

Ratelessness in AWGN channels: In Fig. 1 we compare the achievable throughput as a function of SNR for Raptor codes with 64-QAM modulation versus fixed-rate IEEE 802.11n LDPC codes using belief propagation decoder with soft information and forty full iterations. It is noted the order of signal constellation determines the highest achievable rate of any rateless code. On the other hand, however, using dense constellations renders the rateless code more inefficient since a large number of bits needs to be transmitted per symbols, even if one more bit would be enough to correctly decode. The Raptor code with $k=600$ information bits based on an inner LT code generated using the degree distribution in Raptor RFC 5053 standard [20], with a left regular (degree 3) and right irregular rate 0.96 LDPC pre-code is used. The fixed-rate LDPC codes are simulated on the standard code parameters for 802.11n High-Throughput (HT) mode with 648 codeword length [21], comprising flexible list of modes to provide finely granularity for AMC. An ideal AMC scheme, based on the operations scenario, will choose an LDPC mode that maximizes throughput. However, even in this case a Raptorbased scheme has a finer granularity of throughput over all channel conditions (even in low SNR regime), while each LDPC mode can perform well only in a small range of SNRs. For example, while Raptor and mode 4 have the same throughput at $S N R=7 \mathrm{~dB}$, the Raptor increases throughput by $34 \%$ at $S N R=10 \mathrm{~dB}$.

Comparisons with HARQ schemes: As we mentioned in Section 1, there is an analogy between HARQ and rateless schemes. In particular, HARQ schemes with Incremental

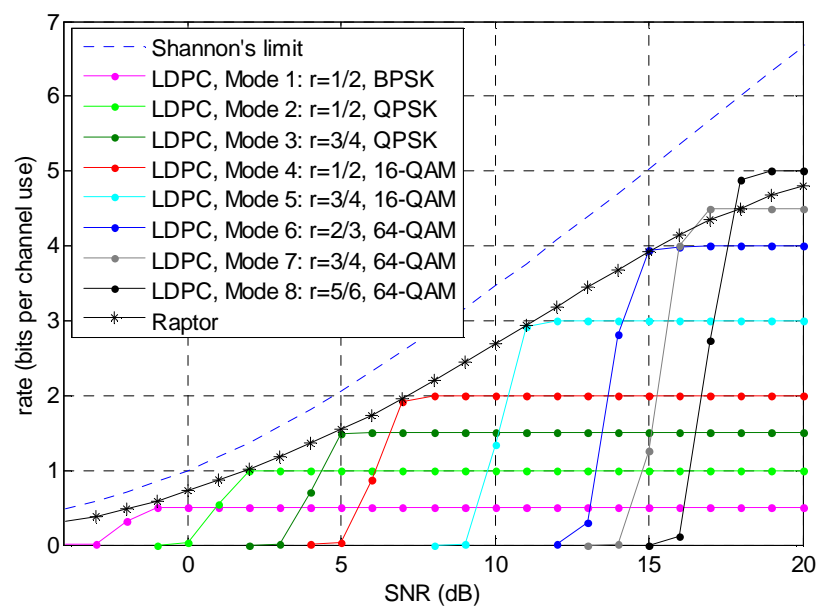

Fig. 1: Achievable rates of Raptor code with 64-QAM and 802.11n LDPC codes over AWGN channel.

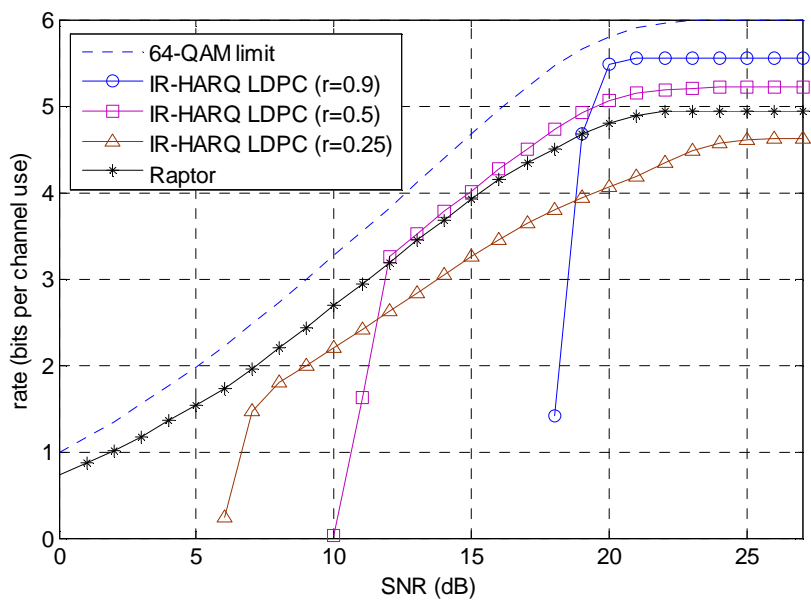

Fig. 2: Achievable rates of Raptor code and IR-HARQ employing punctured LDPCs with 64-QAM over AWGN channel.

Redundancy (IR-HARQ) [7] are very similar to other rateless schemes since they transmit additional symbols until the received information is successfully decoded. Fig. 2 depicts the average throughput performance as a function of SNR for IR-HARQ schemes employing a punctured LDPC (with $k=600$ ) and Raptor codes over AWGN channel with 64-QAM. Here, irregular LDPCs with average left node degree $d_{l} \approx 3$ and right node degree $d_{r}=d_{l} \cdot n /(n-k)$ are used, where $n$ is the codeword length. The Raptor code employs the same structure as in previous simulations, with the same decoding parameter. From Fig 2, we observe that IR-HARQ schemes are not capable of operating over the whole SNR range, and therefore, they need to be applied in conjunction with some form of AMC. We found our results in agreement with the trends in [22]-[24].

Ratelessness in block-fading channels: Fig. 3 shows the same results as in Fig. 1 but for block-fading channels. This emulates the performance in scenarios where an AMC 
cannot know the exact channel conditions and it performs rate adaptation based on the channel statistics. We see that, in such a challenging scenario, the performance difference between an AMC scheme and a rateless code is substantial. This is due to the fact that for a fixed-rate code there exists a fading condition such that probability of outage is non-zero. However, with the rateless property, the outage probability may approach zero in spite of channel conditions since the effective code rate is actually determined by the decoder not the encoder [25], i.e. decoder acknowledges successful decoding and terminates transmission.

Ratelessness in AWGN channels with erasures: Here we investigate the throughput comparison of Raptor versus 802.11n LDPC schemes for the AWGN with symbol erasure probability $p$. This emulates uncertain scenarios in real networks where symbols may be lost due to collisions, abrupt changes in the transmission, random and short-burst interference from internet-of-things (IoT) devices, or even from hardware imperfections (e.g., sampling offset errors). In the LDPC case, we consider an ideal AMC scheme performing mode selection, based on the results of Fig. 1, and being unaware of potential collisions. In particular, we consider: mode 1 for $0 \mathrm{~dB}$, mode 2 for $2 \mathrm{~dB}$, mode 3 for 5 $\mathrm{dB}$, mode 4 for $8 \mathrm{~dB}$, mode 5 for mode $12 \mathrm{~dB}$, mode 6 for $16 \mathrm{~dB}$, mode 7 for $17 \mathrm{~dB}$, and mode 8 for $19 \mathrm{~dB}$ (see Fig. 4). We show performances for symbol erasure probabilities $p=0.01$ (Fig. 5) and $p=0.1$ (Fig. 6). As can be seen, for $p=0.1$, we show that in contrast to AMC, rateless codes are robust to erasures while typical AMC schemes are not.

The reason why the performance of AMC is not degraded smoothly is that different modes have different robustness to erasures due to their different modulation orders and coding rates.

\section{SPATIALLY-MULTIPLEXED (SM)-MIMO SYSTEMS WITH RATELESS PROPERTIES}

Ratelessness in MIMO systems with Spatial Multiplexing: SM-MIMO systems have attracted attention due to their ability to scale link and network capacity (and throughput) by concurrently transmitting different information streams from each antenna [26]. However, the performance of such systems is determined by the ability to reliably detect and decode the transmitted information. Since, in the framework of rateless transmission, most systems perform beliefpropagation decoding, the quality of the "soft-detector", namely the quality of the module that excises reliability (i.e., soft) information of the received bits, will be critical in determining the performance of the rateless system.

In Fig. 7 two soft-detector approaches have been considered. The first is based on the well-known ZeroForcing (ZF) decoder [27] which equalizes the MIMO channel before the soft information calculation takes place. This is one of the less computational intensive solutions but also highly suboptimal. The second approach is based on "Soft-Sphere-Decoder" (SSD) which jointly calculates the

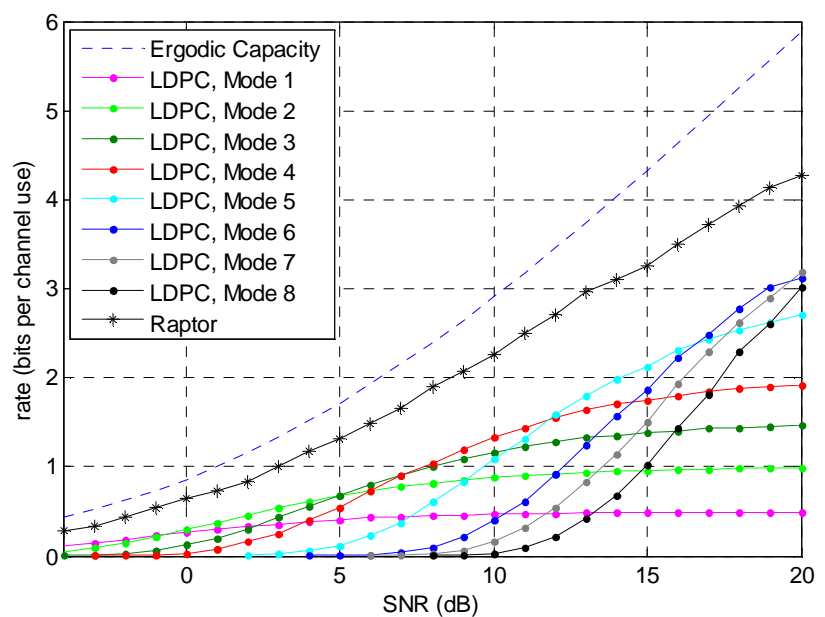

Fig. 3: Achievable rates of Raptor code with 64-QAM and 802.11n LDPC codes in block-fading channel.

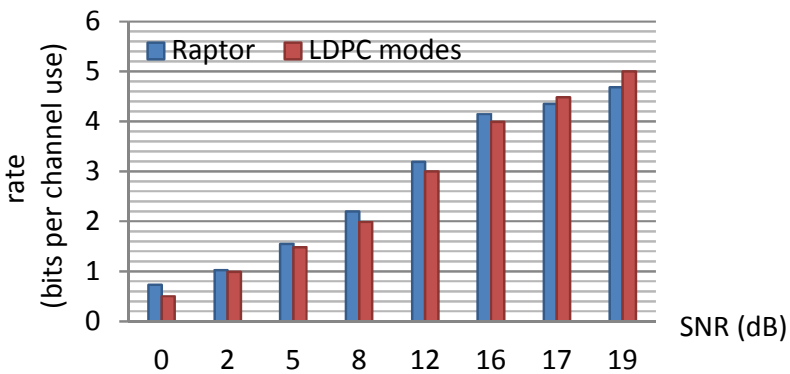

Fig. 4: Achievable rates of Raptor code with 64-QAM over AWGN channel with symbol erasure probability $p=0$.

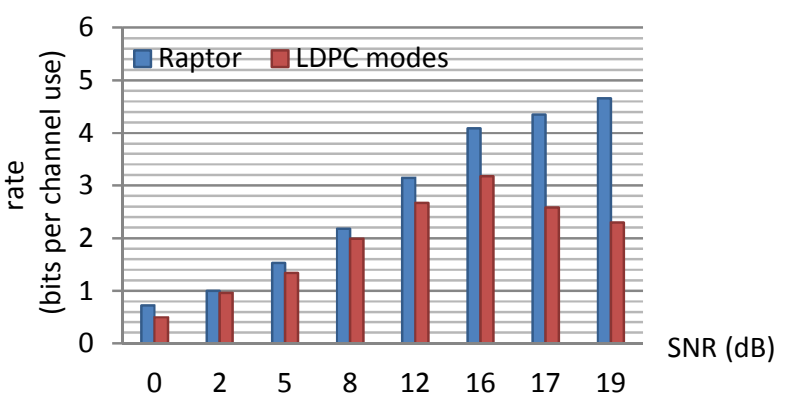

Fig. 5: Achievable rates of Raptor code with 64-QAM over AWGN channel with symbol erasure probability $p=0.01$.

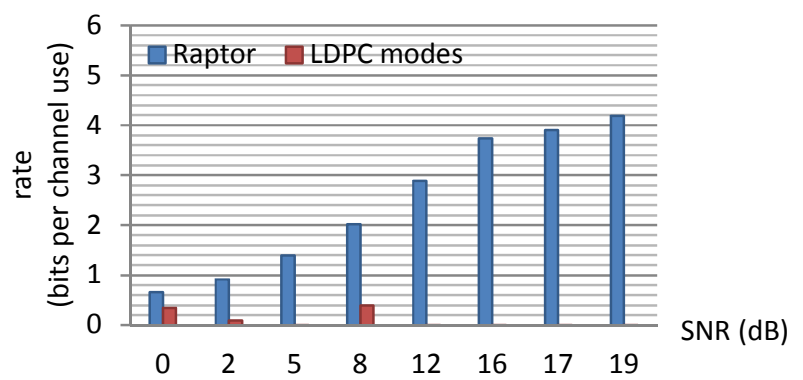

Fig. 6: Achievable rates of Raptor code with 64-QAM over AWGN channel with symbol erasure probability $p=0.1$. 
soft information by transforming its calculation problem into a (multiple) tree search problem. In Fig. 7 we see that the performance of SSD is consistently better, especially in the low SNR regime. It nearly doubles the throughput when compared to ZF. However, the throughput gain comes at the cost of high complexity.

It is known [28],[29] that the complexity of SSD is closely related to the modulation order for two reasons. First, increasing the modulation order will increase the number of nodes of the sphere decoding tree and therefore the corresponding search space. The second reason is that efficient sphere decoder tree search requires a specific ordering in the way they visit the nodes at each level of the tree (i.e., Schnorr-Euchner enumeration [28]), which becomes increasingly complex with the order of the employed modulation [30],[31]. Fig. 8 shows the number of the required visiting nodes (that can determine soft detector's latency) and the number of the required complex multiplications (that can determine power consumption and area). It is shown that the number of required visiting nodes and the number of required complex multiplications substantially increase with the order of the employed QAM modulation and therefore the maximum achievable throughput from the rateless system. To reduce the SSD complexity, promising solutions include the enumeration approaches in [31] and [29]. However, we are still missing practical approaches that can work for high-order constellations (e.g., 64-/256-QAM) and large number of antennas (e.g., $10 \times 10 \mathrm{MIMO}$ ).

In addition, in contrast to single antenna channels, the performance of the corresponding schemes is still far from the capacity limit (see Fig. 7). In order to bridge this gap, iterations are required between the soft-detector and channel decoder [32]. In such scenarios, approaches similar to [33] in combination with the enumeration methods in [30] and [34] can be used to moderate the corresponding complexity and latency requirements.

\section{CONCLUSION}

In this paper, we examined the performance of systems with rateless properties over different channel conditions. We showed that they can result in substantial throughput gains compared to traditional AMC and HARQ approaches, especially in challenging transmission environments. However, processing complexity seems to be one of the main challenges that need to be addressed in order to make such systems practical for future wireless communication systems employing multiple antennas as a way to scale wireless system capacity.

In principle, the gains of rateless codes are based on the assumption that the transmitter can be "immediately" informed to stop transmission if the receiver is able to correctly retrieve the transmitted information. However, this is not feasible. In practice, decoding may take multiple sampling periods, even when the incremental decoding

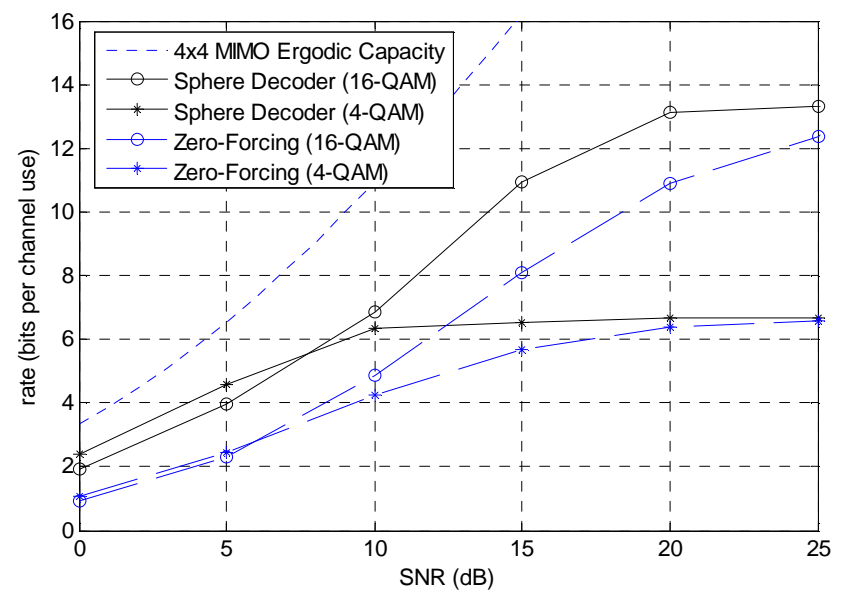

Fig. 7: Achievable rates of Raptor codes with 16-QAM and 4QAM over $4 \times 4$ MIMO channel with ZF and SSD, using belief propagation decoder with fifty iterations.

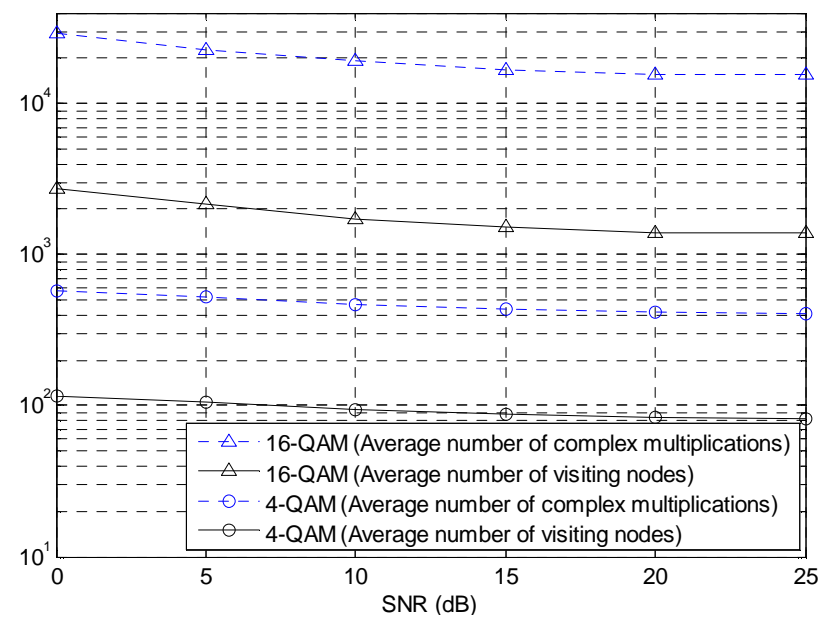

Fig. 8: Average number of visiting nodes and complex multiplications for Raptor codes with 16-QAM and 4-QAM over $4 \times 4$ MIMO channel employing SSD.

approach is applied [35] to reduce the decoding latency. Then, several symbols may need to be transmitted before reattempting decoding, depending on the decoding complexity and the sampling rate of the system. Additional delays can occur for "notifying" the transmitter to stop transmitting [36]. How all these practical aspects can be resolved, and how much of these theoretical gains we can capitalize in practice are open issues to be addressed.

\section{ACKNOWLEDGEMENT}

This work was supported by the Royal Academy of Engineering Award (batch reference 1314-2). 


\section{REFERENCES}

[1] C. Berrou, A. Glavieux, and P. Thitimajshima, "Near Shannon limit error-correcting coding and decoding: turbo codes," in Proc. IEEE ICC, Geneva, Switzerland, May 1993, pp. 1064-1070.

[2] R. G. Gallager, "Low-density parity-check codes," IRE Trans. Inf. Theory, vol. 8, no. 1, pp. 21-28, Jan. 1962.

[3] D. J. C. MacKay, "Good error-correcting codes based on very sparse matrices," IEEE Trans. Inf. Theory, vol. 45, no. 2, pp. 399431, Mar. 1999.

[4] Q. Liu, S. Zhou, and G. B. Giannakis, "Cross-layer combining of adaptive modulation and coding with truncated ARQ over wireless links," IEEE Trans. Wireless Commun., vol. 3, no. 5, pp. 1746-1755, Sep. 2004.

[5] R. A. Comroe and D. J. Costello, "ARQ schemes for data transmission in mobile radio systems," IEEE J. Sel. Areas Commun., vol. 2, no. 4, pp. 472 -481, Jul. 1984.

[6] D. J. Costello, J. Hagenauer, H. Imai, and S. B. Wicker, "Applications of error-control coding," IEEE Trans. Inf. Theory, vol. 44, no. 6, pp. 2531-2560, Oct. 1998.

[7] C. Lott, O. Milenkovic, and E. Soljanin, "Hybrid ARQ: theory, state of the art and future directions," in Proc. IEEE ITW, Solstrand, Norway, Jul. 2007, pp.1-5.

[8] M. Luby, "LT codes," in Proc. IEEE FOCS, Vancouver, Canada, Nov. 2002, pp. 271-280.

[9] A. Shokrollahi, "Raptor codes," in Proc. IEEE ISIT, Chicago, USA, Jun./Jul. 2004, pp. 36.

[10] A. Shokrollahi, "Raptor codes," IEEE Trans. Inf. Theory, vol. 52, no. 6, pp. 2551-2567, Jun. 2006.

[11] O. Etesami and A. Shokrollahi, "Raptor codes on binary memoryless symmetric channels," IEEE Trans. Inf. Theory, vol. 52, no. 5, pp. 2033-2051, May 2006.

[12] R. Palanki and J. S. Yedidia, "Rateless codes on noisy channels," in Proc. IEEE ISIT, Chicago, USA, Jun./Jul. 2004, pp. 38 .

[13] Y. Ma, D. Yuan, and H. Zhang, "Fountain codes and applications to reliable wireless broadcast system," in Proc. IEEE ITW, Chengdu, China, Oct. 2006, pp. 66-70.

[14] J. Perry, P. A. Iannucci, K. Fleming, H. Balakrishnan, and D. Shah, "Spinal codes," in Proc. ACM SIGCOMM, Helsinki, Finland, Aug. 2012, pp. 49-60.

[15] A. Gudipati and S. Katti, "Strider: Automatic rate adaptation and collision handling," in Proc. ACM SIGCOMM, Toronto, Canada, Aug. 2011, pp. 158-169.

[16] Y. Fan, L. Lai, E. Erkip, and H. V. Poor, "Rateless coding for MIMO block fading channels," in Proc. IEEE ISIT, Toronto, Canada, Jul. 2008, pp. 2252-2256.

[17] Y. Fan, L. Lai, E. Erkip, and H. V. Poor, "Rateless coding for MIMO fading channels: performance limits and code construction," IEEE Trans. Wireless Commun., vol. 9, no. 4, pp. 1288-1292, Apr. 2010.

[18] M. M. Shanechi, U. Erez, G. W. Wornell, and K. P. Boyle, "Time-invariant rateless codes for MIMO channels," in Proc. IEEE ISIT, Toronto, Canada, Jul. 2008, pp. 2247-2251.

[19] R. Barron and J. Shapiro, "Design of raptor codes for parallel awgn channels and slow-fading MIMO channels," in Proc. IEEE MILCOM, San Jose, USA, Oct./Nov. 2010, pp. 820-825.
[20] M. Luby, A. Shokrollahi, M. Watson, and T. Stockhammer, "Raptor forward error correction scheme for object delivery," RFC 5053 (Proposed Standard), Oct. 2007.

[21] IEEE Std 802.11n-2009: IEEE Standard for Information technology - Local and metropolitan area networks - Specific requirements - Part 11: Wireless LAN Medium Access Control (MAC) and Physical Layer (PHY) Specifications Amendment 5: Enhancements for Higher Throughput.

[22] N. Bonello, Y. Yang, S. Aissa, and L. Hanzo, "Myths and realities of rateless coding," IEEE Commun. Mag., vol. 49, no. 8, pp. 143-151, Aug. 2011.

[23] E. Soljanin, N. Varnica, and P. Whiting, "Punctured vs rateless codes for Hybrid ARQ," in Proc. IEEE ITW, Punta del Este, Uruguay, Mar. 2006, pp. 155-159.

[24] D. Sejdinovic, V. Ponnampalam, R. J. Piechocki, and A. Doufexi, "The throughput analysis of different IR-HARQ schemes based on fountain codes," in Proc. IEEE WCNC, Las Vegas, USA, Mar./Apr. 2008, pp. 267-272.

[25] N. Bonello, R. Zhang, S. Chen, and L. Hanzo, "Reconfigurable rateless codes," IEEE Trans. Wireless Commun., vol. 8, no. 11, pp. 5592-5600, Nov. 2009.

[26] E. Telatar, "Capacity of multi-antenna Gaussian channels," European Trans. Telecommun., vol. 10, no. 6, pp. 585-595, Nov./Dec. 1999.

[27] C. Wang, E. K. S. Au, R. D. Murch, W. H. Mow, R. S. Cheng, and V. Lau, "On the performance of the MIMO zero-forcing receiver in the presence of channel estimation error," IEEE Trans. Wireless Commun., vol. 6, no. 3, pp. 805-810, Mar. 2007.

[28] C. Studer, A. Burg, and H. Bolcskei, "Soft-output sphere decoding: algorithms and VLSI implementation," IEEE J. Sel. Areas Commun., vol. 26, no. 2, pp. 290-300, Feb. 2008.

[29] K. Nikitopoulos, A. Karachalios, and D. Reisis, "Exact maxlog MAP soft-output sphere decoding via approximate SchnorrEuchner enumeration,” IEEE Trans. Veh. Technol., Aug. 2014.

[30] K. Nikitopoulos, D. Zhang, I. W. Lai, and G. Ascheid, "Complexity-efficient enumeration techniques for soft-input, softoutput sphere decoding," IEEE Commun. Lett., vol. 14, no. 4, pp. 312-314, Apr. 2010.

[31] K. Nikitopoulos, J. Zhou, B. Congdon, and K. Jamieson, "Geosphere: consistently turning MIMO capacity into throughput," in Proc. ACM SIGCOMM, Chicago, USA, Aug. 2014, pp. 631642.

[32] B. M. Hochwald and S. T. Brink, "Achieving near-capacity on a multiple-antenna channel," IEEE Trans. Commun., vol. 51, no. 3, pp. 389-399, Mar. 2003.

[33] K. Nikitopoulos and G. Ascheid, "Approximate MIMO iterative processing with adjustable complexity requirements," IEEE Trans. Veh. Technol., vol. 61, no. 2, pp. 639-650, Feb. 2012.

[34] C. H. Liao, I. W. Lai, K. Nikitopoulos, F. Borlenghi, D. Kammler, M. Witte, D. Zhang, T. D. Chiueh, G. Ascheid, and H. Meyr, "Combining orthogonalized partial metrics: efficient enumeration for soft-input sphere decoder," in Proc. IEEE PIMRC, Tokyo, Japan, Sep. 2009, pp. 1287-1291.

[35] K. Hu, J. Castura, and Y. Mao, "Performance-complexity tradeoffs of Raptor codes over Gaussian channels," IEEE Commun. Lett., vol. 11, no. 4, pp. 343-345, Apr. 2007.

[36] P. Iannucci, J. Perry, H. Balakrishnan, and D. Shah, "No symbol left behind: a link-layer protocol for rateless codes," in Proc. ACM MobiCom, Istanbul, Turkey, Aug. 2012, pp. 17-27. 